\title{
Supplementary Information: Sub-Threshold
}

\section{Continuum Conductance Change In $\mathrm{NbO} \mathrm{Pt}$}

\section{Memristor Interfaces}

Curtis J. O'Kelly ${ }^{1 *}$, Heba N. M. Abunahla ${ }^{1}$, Maguy Abi Jaoude ${ }^{2}$, Dirar Homouz ${ }^{2}$ and Baker

Mohammad $^{1 *}$.

${ }^{1}$ Department of Electrical and Computer Engineering

${ }^{2}$ Department of Applied Mathematics and Sciences

Khalifa University of Science, Technology and Research, Abu Dhabi, UAE

Corresponding Authors

* curtis.okelly@kustar.ac.ae + +971(0)2 5018492

* baker.mohammad@kustar.ac.ae $\quad$ +971 (0)2 5018513 
(a)

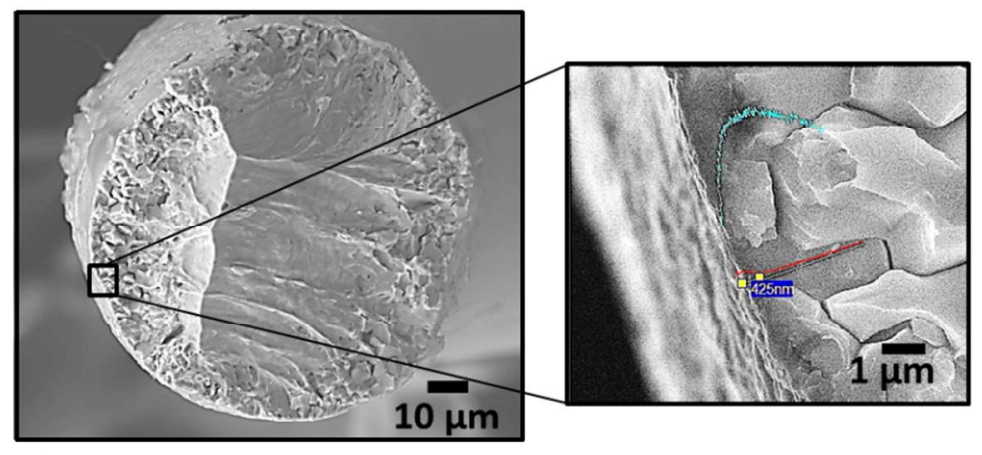

(b)

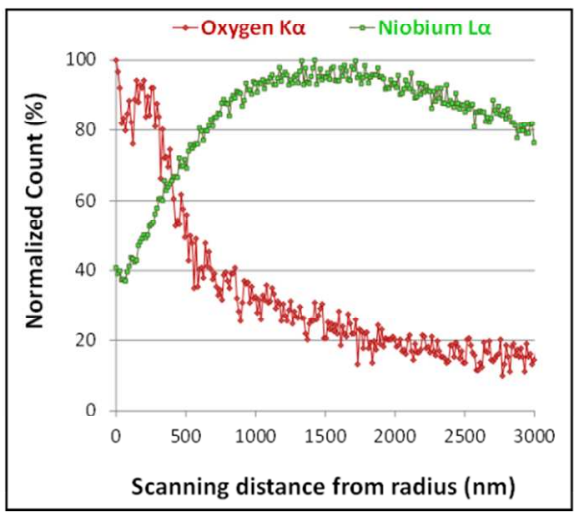

Supplementary figure S1. EDX analysis of a cross section of NbO core shell wire showing the

Oxygen and Niobium signals and how they change with depth across the skin of the wire.

(a)

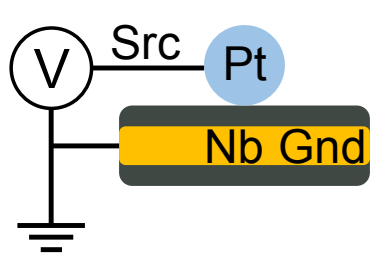

(b)

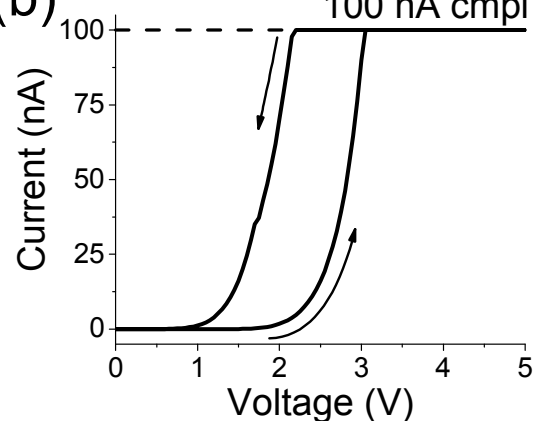

(c)

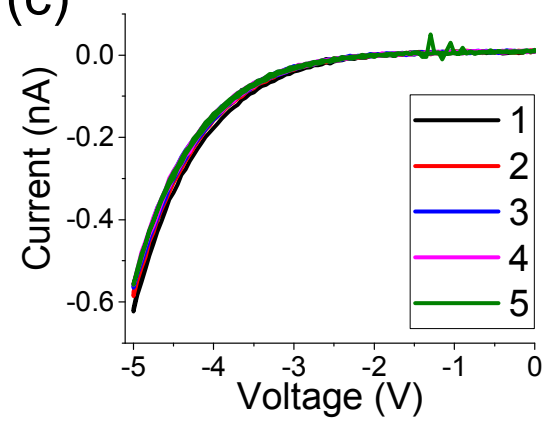

Supplementary figure S2. I-V characteristics of the device in the standard orientation (a). (b) Forward biasing the Pt reveals the current hysteresis. (c) No current hysteresis or current growth is observed when negative polarity is applied to the Pt electrode even after 5 sweeps. 

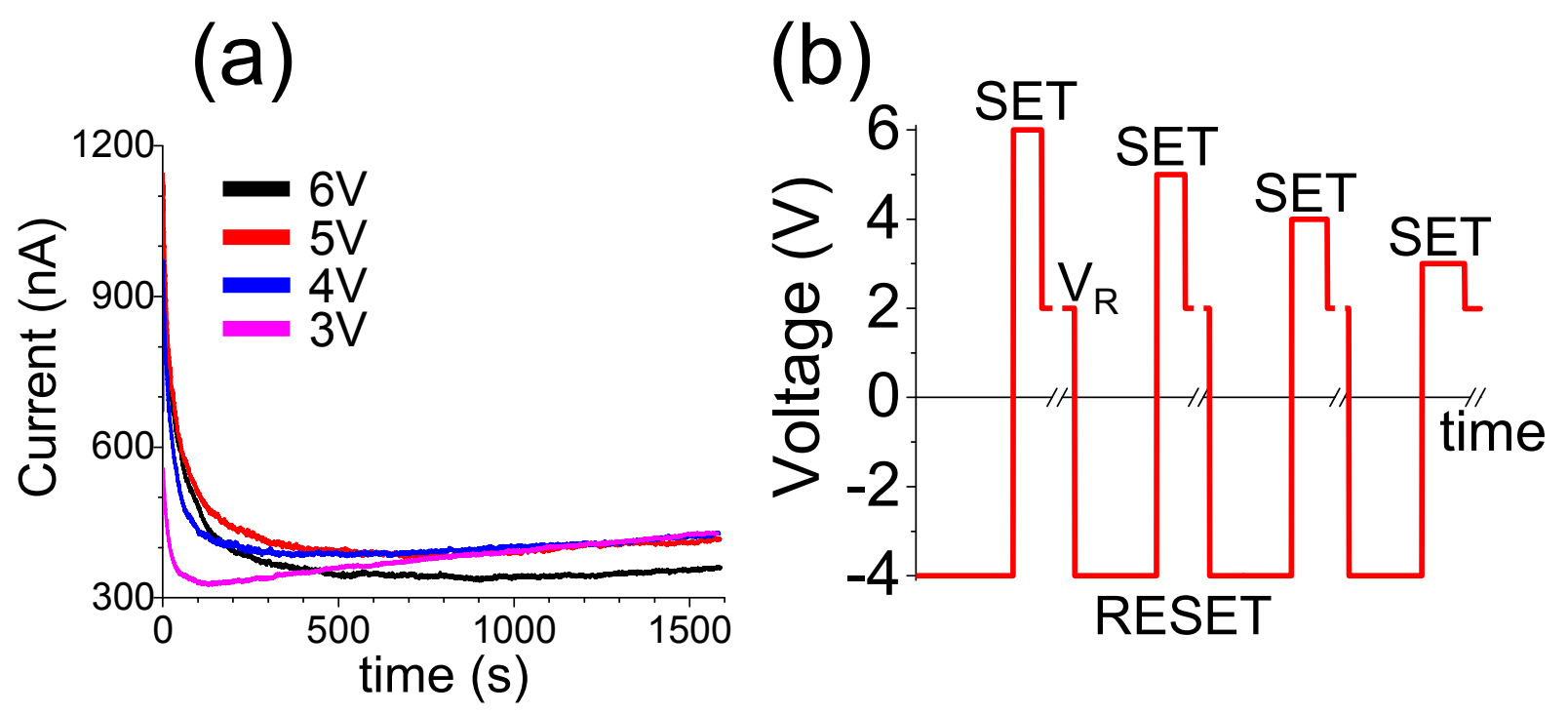

Supplementary figure S3. Equal flux pulses with different voltage amplitudes are applied to the device with a reset step in between each. The conductivity approaches similar levels after the 5, 4 and $3 \mathrm{~V}$ equal flux pulse but the $6 \mathrm{~V}$ pulse remains at a lower conductivity. This may be related to the difficulty in finding a common reference point or condition
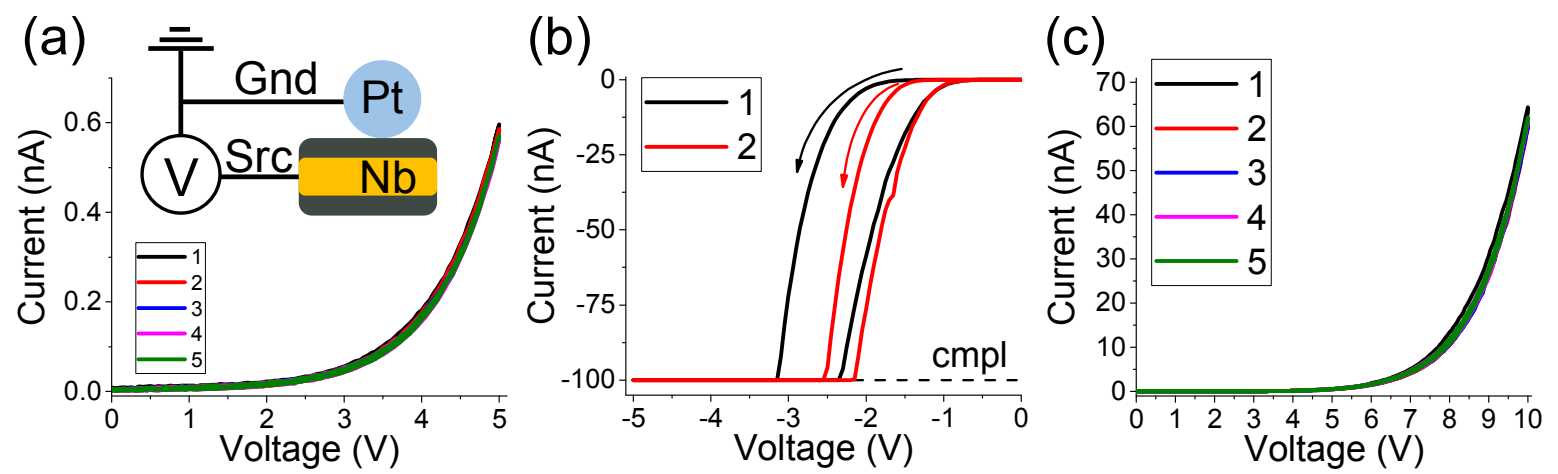

Supplementary figure S4. (a) Swapping the electrodes so that the $\mathrm{Nb}$ electrode is the source reveals the same I-V behavior but in opposite direction. (b) Negative polarity applied at the $\mathrm{Nb}$ electrode produces the same hysteresis and conductivity change as observed previously. (c) Applying voltage sweeps up to $10 \mathrm{~V}$ does not alter the conductivity of the $\mathrm{Nb} / \mathrm{NbO}$ interface. 


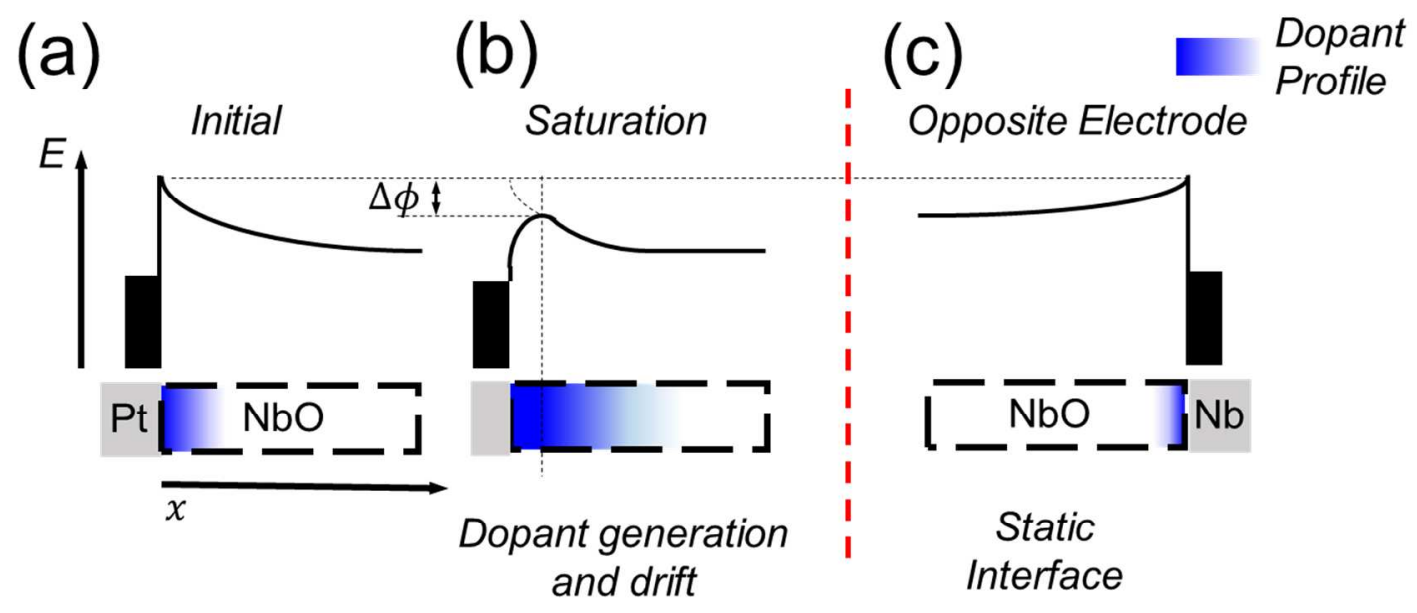

Supplementary figure S5. (a) Schematic depicting the initial Pt/NbO interface. (b) The Pt/NbO interface upon saturation. Dopants are generated at the metal semiconductor interface and drift/diffuse through the interface lowering the barrier for carrier extraction. (c) At the $\mathrm{NbO} / \mathrm{Nb}$ interface dopants are not generated and it remains static with unchanging electrical properties. 